\title{
Two-state behaviour of Kondo trimers
}

\author{
M. E. Torio, ${ }^{1}$ K. Hallberg, ${ }^{2}$ and C. R. Proetto ${ }^{2}$ \\ ${ }^{1}$ Instituto de Física Rosario, CONICET-UNR, \\ Bv 27 de Febrero 210 bis, (2000) Rosario, Argentina \\ ${ }^{2}$ Centro Atómico Bariloche and Instituto Balseiro, CNEA \\ 8400 Bariloche, Argentina
}

\begin{abstract}
The electronic properties and spectroscopic features of a magnetic trimer with a Kondo-like coupling to a non-magnetic metallic substrate are analyzed at zero temperature. The substrate density of states is depressed in the trimer neighbourhood, being exactly zero at the substrate chemical potential. The size of the resonance strongly depends on the magnetic state of the trimer, and exhibits a two-state behavior. The geometrical dependence of these results agree qualitatively with recent experiments and could be reproduced in a triangular quantum dot arrangement.
\end{abstract}

Keywords:

The combination of the Scanning Tunneling Microscope (STM), which can be used as a "tool" to build and characterize atomically-precise structures, and the availability of high-quality clean metallic surfaces has provided a unique opportunity to investigate the chemical and physical properties of deposited atoms, molecules, and clusters with unprecedented resolution. It has been natural then that much of the research concentrates on one of the old paradigms of condensed-matter physics: the behavior of magnetic impurities immersed in an otherwise non-magnetic matrix. Ground-breaking experiments have been reported on the properties of magnetic monomers [1] and dimers 2], quantum-confinement of surface two-dimensional electron gases by a "corral" of STM-positioned magnetic atoms 3], and "molecule cascade" devices [4], with potential for quantum computing. In most of these examples, it has become evident that at low-temperatures the physics of magnetic impurities on the metallic surface is dominated by the so-called Kondo effect. Historically, the Kondo effect was introduced more than forty years ago to explain the resistivity minimum for decreasing temperatures observed in metallic matrices with a minute fraction of magnetic impurities. [5] Additional interest in the Kondo effect arose recently from the semiconductor nano-oriented community, after realization $7,[8]$ that a quantum dot with two attached conducting leads behaves in many aspects as a magnetic atom in a metal. 9, 10]

The aim of this work is the theoretical study of a magnetic trimer in contact with a metallic substrate. The trimer geometry is very interesting, due to the fact that it is the simplest geometrical arrangement (including monomers and dimers) which displays magnetic frustration among the three magnetic moments for the case of antiferromagnetic interactions 11. The main result from our work is that as a consequence of the interplay between the trimer-metal Kondo interaction which tries to stabilize the trimer in a high-spin state, and the intratrimer antiferromagnetic interactions which try of stabilize a low-spin trimer state, the whole system exhibits

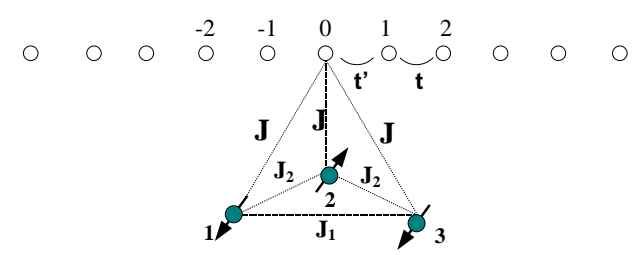

FIG. 1: Schematic diagram of the system considered: an AF spin trimer Kondo-coupled to a non-interacting chain

a two-state spectroscopic behavior that depends on the geometrical arrangement of the triangle (whether it is an equilateral or isosceles triangle) and on the interaction parameters.

The model employed in the calculation consists of two semi-infinite non-interacting tight-binding (TB) chains connected to a central site (also non-interacting). This central site is in turn Kondo-coupled to three magnetic impurities, the trimer.The Hamiltonian reads:

$$
H=H_{c}+H_{c-t}+H_{t},
$$

where $H_{c}$ is the Hamiltonian of two semi-infinite chains,

$$
\begin{aligned}
H_{c} & =-\sum_{j \leq 0, \sigma} t_{j-1}\left(c_{j \sigma}^{\dagger} c_{j-1 \sigma}+\text { h.c. }\right) \\
& -\sum_{j \geq 0, \sigma} t_{j+1}\left(c_{j \sigma}^{\dagger} c_{j+1 \sigma}+\text { h.c. }\right) ;
\end{aligned}
$$

$H_{c-t}$ couples the chains to the trimer and can be written as

$$
H_{c-t}=\sum_{\alpha, \sigma} t_{\alpha}^{\prime \prime}\left(c_{0 \sigma}^{\dagger} d_{\alpha \sigma}+\text { h.c. }\right)
$$

and $H_{t}$ is the trimer Hamiltonian

$$
H_{t}=\sum_{\alpha \neq \beta, \sigma} t_{\alpha \beta} d_{\alpha \sigma}^{\dagger} d_{\beta \sigma}+\sum_{\alpha}\left(\varepsilon_{0} n_{\alpha}+U n_{\alpha \uparrow} n_{\alpha \downarrow}\right) .
$$

In the equations above, $t_{j}=t=1$ for $j \leq-1, j \geq 1$, while $t_{-1}=t_{1}=t^{\prime}=1, \alpha, \beta=1,2,3$ (the trimer sites). 
$c_{j \sigma}\left(c_{j \sigma}^{\dagger}\right)$ corresponds to the destruction (creation) operator for one electron at the chain site $j$ with spin $\sigma$ $(=\uparrow, \downarrow)$, while $d_{\alpha \sigma}\left(d_{\alpha \sigma}^{\dagger}\right)$ is a destruction (creation) operator for electrons at the trimer sites. $n_{\alpha \sigma}=d_{\alpha \sigma}^{\dagger} d_{\alpha \sigma}$, $n_{\alpha}=\sum_{\sigma} n_{\alpha \sigma}$, and $U>0$. If $t_{\alpha}^{\prime \prime}=0$ for $\alpha=1,2,3$, $H_{c-t}=0$ and the whole system becomes the sum of two uncoupled subsystems: the tight-binding chain and the trimer. In the Kondo regime it is feasible to simplify the Hamiltonian above by performing a Schrieffer-Wolff (SW) canonical transformation of the second and third terms in Eq.(1), which projects out of the Hilbert space states where the trimer sites are either empty or double occupied. Keeping only the most relevant terms, the result of the transformation for these terms is (see Fig. 1)

$$
H_{c-t}+H_{t}=\sum_{\alpha} \bar{J}_{\alpha}\left(\mathbf{s}_{0} \cdot \mathbf{S}_{\alpha}\right)+\sum_{\alpha \neq \beta} \bar{J}_{\alpha \beta}\left(\mathbf{S}_{\alpha} \cdot \mathbf{S}_{\beta}\right) .
$$

The first term in Eq.(5) represents an $s$ - $d$ interaction among the trimer spins and the central site of the chain; the second term is a Heisenberg interaction among the spins located at each of the trimer sites. For the symmetric case $2 \varepsilon_{0}+U=0$ considered in the present work, $\bar{J}_{\alpha}=8\left|t_{\alpha}^{\prime \prime}\right|^{2} / U, \bar{J}_{\alpha \beta}=8\left|t_{\alpha \beta}\right|^{2} / U$. As $U>0$, both $\bar{J}_{\alpha}$ and $\bar{J}_{\alpha \beta}$ are positive, corresponding to antiferromagnetic interactions in Eq.(5). Unless otherwise stated, we will assume in this work a symmetric coupling configuration: $\bar{J}_{1}=\bar{J}_{2}=\bar{J}_{3}=J$, and $\bar{J}_{13}=J_{1}, \bar{J}_{12}=$ $\bar{J}_{23}=J_{2}$. Numerical calculations have been performed for both Anderson-like (Eqs.(2-4)) and Kondo/Heisenberglike models (Eq.(2),(5)), but results will be shown only for the latter. Results considering Anderson impurities in the SW limit present a qualitatively similar physical behaviour.

Since the possible trimer states will play a relevant role in the following discussions, we will write down here its eigenvectors and eigenvalues. From Eq.(5) we have $H_{t}=$ $J_{1} \mathbf{S}_{1} \cdot \mathbf{S}_{3}+J_{2}\left(\mathbf{S}_{1} \cdot \mathbf{S}_{2}+\mathbf{S}_{2} \cdot \mathbf{S}_{3}\right)$. If $J_{1}=J_{2}$ the trimer is in the equilateral (E) configuration, whereas if $J_{1} \neq J_{2}$ the trimer is in the isosceles (I) configuration. The Hilbert space of $H_{t}$ comprises eight states: a quartet, and two doublets. The quartet $(Q)$ eigenstates each one with total spin $3 / 2$, are given in the real space representation by: $|Q, 3 / 2\rangle=|\uparrow \uparrow \uparrow\rangle,|Q, 1 / 2\rangle=(|\uparrow \uparrow \downarrow\rangle+|\uparrow \downarrow \uparrow\rangle+|\downarrow \uparrow \uparrow\rangle) / \sqrt{3}$, while $|Q,-3 / 2\rangle$ and $|Q,-1 / 2\rangle$ are obtained from $|Q, 3 / 2\rangle$ and $|Q, 1 / 2\rangle$ under the change $\uparrow \longleftrightarrow \downarrow$, respectively. It can be checked that $H_{t}\left|Q, S_{z}\right\rangle=\left(J_{1}+2 J_{2}\right) / 4\left|Q, S_{z}\right\rangle$, with $S_{z}= \pm 3 / 2, \pm 1 / 2$. The eigenstates of the two doublets $\left(D\right.$ and $\left.D^{\prime}\right)$, each one with total spin $1 / 2$ are: $|D, 1 / 2\rangle=$ $(|\uparrow \uparrow \downarrow\rangle-|\downarrow \uparrow \uparrow\rangle) / \sqrt{2},\left|D^{\prime}, 1 / 2\right\rangle=(|\uparrow \uparrow \downarrow\rangle-2|\uparrow \downarrow \uparrow\rangle+$ $|\downarrow \uparrow \uparrow\rangle) / \sqrt{3}$, with $|D,-1 / 2\rangle$ and $\left|D^{\prime},-1 / 2\right\rangle$ obtained again by switching up and down spins. $H_{t}\left|D, S_{z}\right\rangle=$ $\left(-3 J_{1} / 4\right)\left|D, S_{z}\right\rangle, H_{t}\left|D^{\prime}, S_{z}\right\rangle=\left(J_{1} / 4-J_{2}\right)\left|D^{\prime}, S_{z}\right\rangle$, with $S_{z}= \pm 1 / 2$. For $J_{1}, J_{2}>0$, as obtained from the SW transformation, the ground state (GS) of the isolated

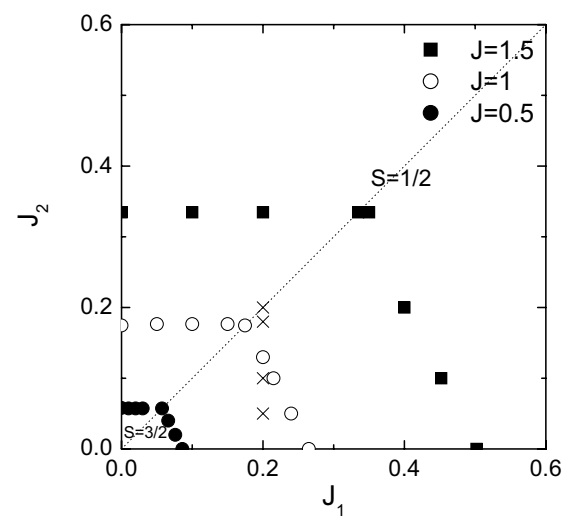

FIG. 2: Boundaries between the strong (large spin) and weak (low spin) Kondo states for three different values of $J$. For each boundary, the large (small) $J_{1}, J_{2}$ values correspond to the low (high) spin values, $S=1 / 2$ and $3 / 2$ respectively. The crosses mark the positions where the local DOS has been calculated (Fig. 4).

trimer as a function of $J_{2}$ changes from $\left|D, S_{z}\right\rangle$ to $\left|D^{\prime}, S_{z}\right\rangle$ at $J_{2}=J_{1}, i$. $e$. the equilateral case. It is important to note that even for the coupled chain-trimer Hamiltonian, Eq.(5), the total spin of the trimer remains a good quantum number of the whole system and this is why we can characterize the states in the complete system by the total spin of the trimer.

In Fig. 2 we present the phase diagram of the trimer coupled to the chain, showing the critical lines separating both total spin ground states in the trimer $(3 / 2$ and $1 / 2)$, for different values of $J$ and $N=13$, the total length of the tight binding chain. The phases were obtained by comparing ground state energies calculated using exact diagonalization. For small interaction parameters the system is in the high-spin state (below each curve) and for large parameters it is in the low-spin state (above the curves). The high spin trimer state is increasingly Kondo-stabilized as $J$ increases. The critical boundary has also a different behaviour at the left or right of the equilateral line $J_{1}=J_{2}$. To the left of this line, the boundary is horizontal, i.e. for a given value of $J$, the critical value of $J_{2}$ (defined as $J_{2}^{c}$ ) is constant for a large range of $J_{1}$. In this region the transition occurs between the total spin trimer states $|Q\rangle$ and $\left|D^{\prime}\right\rangle$ when increasing $J_{2}$ for fixed $J_{1}$ (see case (b) below Eq. (8)). To the right of the equilateral line, the boundary is linear and, increasing $J_{2}$ similarly as above, the system evolves from the $|Q\rangle$ trimer state to the second doublet state $|D\rangle$. For a larger value of $J_{2}$ (not shown), it then turns finally into state $\left|D^{\prime}\right\rangle$ (case (a) explained below Eq. 8)).

The scaling behaviour of this phase diagram is shown in Fig. 3, where we present two magnitudes which serve as useful characterizations of the whole system. In Fig. 3 (a) we plot the Kondo energy $E_{K}(\mathbf{S}) \equiv E_{G S}(N, \mathbf{S})-$ $\left[E_{\text {chain }}(N)+E_{\text {trimer }}(\mathbf{S})\right]$. Note that by substracting $E_{\text {chain }}(N)$ (defined as the energy of the tight-binding 


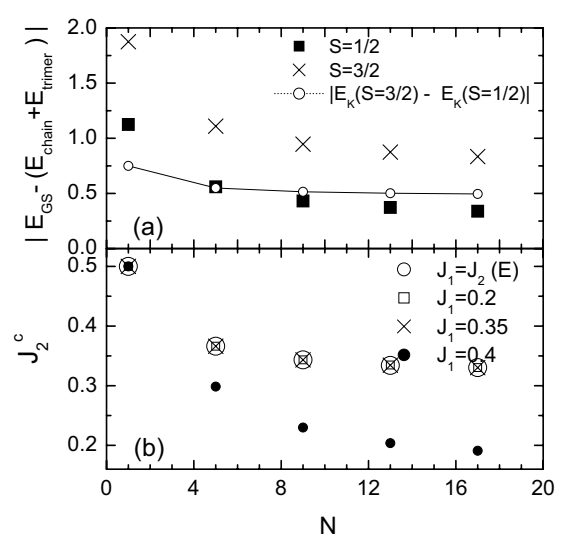

FIG. 3: Scaling behaviour of a) Absolute value of the Kondo energy $E_{K}(\mathbf{S})$ for both spin states of the trimer and their difference (here $\left.J_{1}=0.35\right)$; b) the critical $J_{2}\left(J_{2}^{c}\right)$ value marking the transition between both spin states, for several values of the parameters. In both panels the high spin (low spin) state corresponds to $J_{2}<J_{2}^{c}\left(J_{2}>J_{2}^{c}\right)$ and $J=1.5$.

chain with $J=0$ ) and $E_{\text {trimer }}(\mathbf{S})$, what is left is the non-trivial component of the GS energy related to the Kondo coupling among the magnetic sites of the trimer and the chain. Accordingly, we denote this energy as $E_{K}(\mathbf{S})$, with $\mathbf{S}$ representing the total spin of the trimer. As can be seen, the Kondo energy for the large spin state is larger in magnitude than for the low spin state. In Fig. $3(\mathrm{~b})$ we show the scaling behavior of $\left(J_{2}^{c}\right)$. For values of $J_{1}$ smaller than or equal to the equilateral case, the value of $J_{2}^{c}$ remains the same (see also Fig. 2), whereas for the opposite case, $J_{2}^{c}$ is smaller (full dots).

In order to understand the results displayed in Figs. 2 and 3 it is quite instructive to solve the case with one conduction site, $N=1, i$. e e the tetrahedron (considering one conduction electron). Noting that $E_{\text {chain }}(N=$ 1) $=0$, and considering that the trimer could be either in a state with total spin $3 / 2$ or $1 / 2$, we have three possible states for $E_{G S}(1, \mathbf{S})$ :

$$
\begin{aligned}
E_{G S}(1, Q) & =\frac{1}{4}\left(J_{1}+2 J_{2}\right)-\frac{5}{4} J, \\
E_{G S}(1, D) & =-\frac{3}{4} J_{1}-\frac{3}{4} J, \\
E_{G S}\left(1, D^{\prime}\right) & =\frac{1}{4}\left(J_{1}-4 J_{2}\right)-\frac{3}{4} J
\end{aligned}
$$

The second term which appears on the r.h.s. of Eqs. (6-8), corresponds either to $E_{K}(3 / 2)=-5 J / 4$, or $E_{K}(1 / 2)=-3 J / 4$. These are just the binding energies corresponding to the $s-d$ (Kondo) interaction term of Eq.(5), for the case of a localized magnetic moment with total spin $1 / 2(-3 J / 4)$ or $3 / 2(-5 J / 4)$. For $J=1.5$, $E_{K}(3 / 2)=-1.875, E_{K}(1 / 2)=-1.125$, corresponding to the two $N=1$ points in Fig. 3a. As $N$ increases, both $E_{K}(3 / 2)$ and $E_{K}(1 / 2)$ evolve smoothly and their difference is nearly converged for $N=17$ (Fig. 3a). From
Eqs. (6-8) we can calculate $J_{2}^{c}$ for $N=1$, and qualitatively follow its evolution with $N$. For fixed values of $J$ and $J_{1}$, two scenarios for the GS are possible for increasing values of $J_{2}$ : a) $E_{G S}(Q) \rightarrow E_{G S}(D) \rightarrow E_{G S}\left(D^{\prime}\right)$. From $E_{G S}(Q)=E_{G S}(D)$ we obtain $J_{2}^{c}=J-2 J_{1}$; b) $E_{G S}(Q) \rightarrow E_{G S}\left(D^{\prime}\right)$. From $E_{G S}(Q)=E_{G S}\left(D^{\prime}\right)$ we obtain $J_{2}^{c}=J / 3$. The $J_{2}^{c}$ corresponding to the cases $J_{1}=J_{2}$, (i.e. always in the equilateral configuration), $J_{1}=0.2$, and 0.35 all fall in case b) and the three curves fall onto a single curve (Fig. $3 \mathrm{~b}$ ). For $J_{1}=0.4$, however, case a) is pertinent, and $J_{2}^{c}$ displays a distinct behavior. Both kinds of transitions can be seen also in a different behaviour of the boundary lines in Fig. 2.

The fact that the total spin of the trimer presents two different values, leads to a well defined two-state pattern in the Kondo behaviour of the system. As we showed before with the energies, the Kondo energy is stronger for the high spin state. This can be clearly seen in the local density of states (DOS) at the central site, where it should present a dip when the Kondo efect is operative 12, 13 .

In Fig. 4 the local density of states at the central site of the chain is shown. To obtain the density of states $\rho_{\sigma}(\omega)$ we use a combined method. In the first place we consider an open finite cluster of $N$ sites $(N=13$ in our case) which includes the trimer. This is diagonalized using the exact diagonalization Lanczos technique 14]. We then proceed to embed the cluster in an external reservoir of electrons, which fixes the Fermi level of the system, attaching two semi-infinite leads to its right and left 15 . This is done by calculating the one-particle Green function $\hat{G}$ of the whole system within the chain approximation of a cumulant expansion [16] for the dressed propagators. This leads to the Dyson equation $\hat{G}=\hat{g}+\hat{T} \hat{G}$, where $\hat{g}$ is the cluster Green function obtained by the Lanczos method. The density of states is obtained from $\hat{G}$.

As a comparison, we have also included in Fig. 4 the LDOS of the isolated non-interacting chain, corresponding to the case $J=0$ in our model. A profound dip appears in the LDOS of the chain interacting with the trimer, with the LDOS being exactly zero at the Fermi level of the TB chain $(\omega=0)$. Quite generally, dips of this type arise each time a system with a continuous spectrum (i.e. the chain), interacts with a system with a discrete spectrum (i.e. the trimer) 13. While the original discussion of Fano only addressed the case where the discrete spectrum corresponds to a non-interacting system, his analysis has been generalized to the case of discrete many-body peaks interacting with a featureless continuum spectrum[1]. The resulting dips in the DOS are generally termed "Fano resonances". Two types of Fano resonances are observed in Fig. 4. The broad Fano resonance is associated with the trimer in the state with total spin $3 / 2$ and its width is given by $E_{K}(3 / 2)$. The narrow Fano resonance is associated with the trimer in the state 


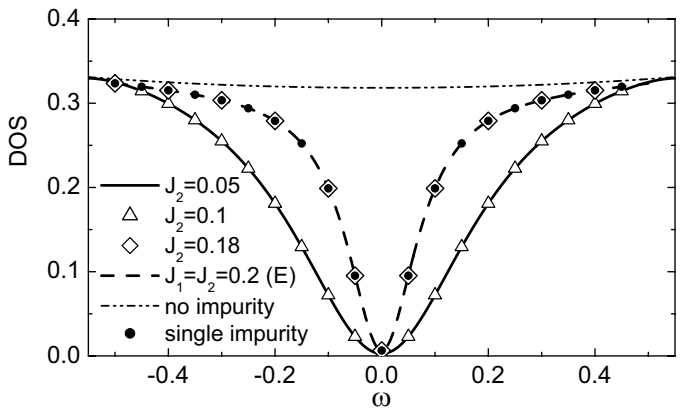

FIG. 4: Local density of states at the central site of the chain for $J_{1}=0.2, J=1$ and several values of $J_{2}$, showing the two-state behaviour of the Kondo effect. Also shown is the $J=0$ case (free chain) where no Kondo dip is present and the single impurity case. For these parameters $T_{K}(E)<T_{K}(I)$.

with total spin $1 / 2$, and its width is given by $E_{K}(1 / 2)$. Two important features should be noticed from Fig. 4: i) the size (width) of the dip only depends on the total spin of the trimer, and not on the particular values of $J_{1}$ and $J_{2}$, as long as the trimer total spin remains invariant. This leads to the bimodal distribution of Fano resonances showed in Fig. 4; ii) the case $J_{1}=J_{2}=0.2$ corresponds to the trimer in the equilateral configuration, with total spin $1 / 2$ and a narrow resonance. For a small reduction in $J_{2}\left(J_{2}=0.1\right)$, the trimer is driven to an isosceles configuration, with an increase of its Kondo energy and the corresponding spectral feature.

Recent experimental results by Jamneala et. al., 17] investigating the local behavior of single triangular $\mathrm{Cr}$ trimers deposited on the surface of gold show that $\mathrm{Cr}$ trimers can be reversibly switched between two distintic electronic states. According to their structural and spectroscopic characterization, one of the two "switching states" corresponds to the "I" configuration which, by using the STM $d I / d V$ spectroscopy, reflects a lowenergy Kondo response (Fano resonance). The second "switching state" was characterized as the trimer in the "E" configuration, for which no low-energy feature was discernible. Our numerical results, based on a simplified model where the hibridization to a single channel is considered, could shed light on these experimental results. It captures the essential physics of this experiment and reproduces its main gross experimental features: the two-state spectroscopic behavior, and the fact that $T_{K}(I)=E_{K}(3 / 2)>T_{K}(E)=E_{K}(1 / 2)$, as displayed in Fig. 4. For any $T$ such that $T_{K}(I)>T>T_{K}(E)$, the narrow Fano resonance associated to the "E" configuration will be lost, as well as the single impurity case, as experimentally observed. The "I" configuration will exhibit, on the contrary, a discernible Fano resonance. This behaviour is obtained when all the antiferromagnetic parameters $J, J_{1}$ and $J_{2}$ are of the same relative order of magnitude 2], where the magnitude is a fraction of an $\mathrm{eV}$. In addition, the isosceles state is obtained from the "E" configuration, as in the experiment, by slightly reducing the value of $J_{2}$, i.e. separating atom number 2 . Our results also agree qualitatively with recent results based on Quantum Monte Carlo calculations 18.

Previous calculations on similar models reach contradictory conclusions [18, 19, 20, 21] finding that the visible Kondo effect corresponds to either equilateral or isosceles configurations. In 20, 21], calculations based on approximate variational and $a b$ initio methods, restricted the Hilbert subspace to the one with a Kondo singlet in the "trimer+conduction state" (states with energies given by Eqs. 7 and 8), and missed the "ferromagnetic" state of the trimer, driven by the Kondo interaction (state with energy given by Eq. 6). Accordingly, the two-state behavior is not present in their results, and moreover, both obtain that $T_{K}(E)>T_{K}(I)$, which does not agree with the experimental interpretation.

This system is an ideal scenario for the experimental observation of a quantum critical point between two states with different quantum numbers and its analysis with finite temperature could lead to interesting physics 22]. The rising activity in experimental and theoretical studies of triple quantum dots 23] is due to the interesting physics that can be obtained in these systems. The feasible construction of a trimer of quantum dots coupled to leads which can be tuned between different quantum states, could lead to clean and controlable results. Conductance measurements performed in such a system could detect between distinct spin states in the trimer and serve as a readout method.

In conclusion, we have obtained a two-state behaviour in a system consisting of a spin $\mathrm{S}=1 / 2$ trimer Kondocoupled to a non-interacting chain. This simplified model explains qualitatively the experimental findings of $\mathrm{Cr}$ trimers deposited on the surface of $\mathrm{Au}$, where, at a certain temperature, a Fano dip was found for the isosceles configuration, but was absent for the equilateral state, indicating a possible lower Kondo temperature in this latter case, as obtained here. Moreover, such a system, experimentally realizable as a triangular quantum dot device, could be used to measure the total spin state of the timer adatom.

The authors acknowledge CONICET for support under grant PIP 0473/98 and ANPCyT under PICT97 03-00121-02152.

[1] V. Madhavan, W. Chen, T. Jamneala, M. F. Crommie, and N. S. Wingreen, Science 280, 567 (1998); J. Li, WD Schneider, R. Berndt, and B. Delley, Phys. Rev. Lett. 80, 2893 (1998).

[2] W. Chen, T. Jamneala, V. Madhavan, and M. F. Crommie, Phys. Rev. B 60, R8529 (1999). 
[3] H. C. Manoharan et al., Nature (London) 403, 512 (2000).

[4] A. Heinrich et al, Science 298, 1381 (2002)

[5] J. Kondo, in Solid State Physics 23, 183 (1969).

[6] A. C. Hewson, in The Kondo Problem to Heavy Fermions (Cambridge University Press, Cambridge, England, 1993).

[7] L. I. Glazman and M. E. Raikh, JETP Lett. 47, 452 (1988).

[8] T. K. Ng and P. A. Lee, Phys. Rev. Lett. 61, 1768 (1988).

[9] D. Goldhaber-Gordon et al., Nature 391, 156 (1998).

[10] S. M. Cronenwet, T. H. Oosterkamp, and L. P. Kouwenhoven, Science 281, 540 (1998).

[11] K. Ingersent, A. Ludwig and I. Affleck, cond-mat/0505303

[12] M. E. Torio, K. Hallberg, A. H. Ceccatto, and C. R. Proetto, Phys. Rev. B 65, 085302 (2002); G. Lara et al., cond-mat/0411661 M. Sato et al., Phys. Rev. Lett. 95, 066801 (2005); K. Kobayashi et al., Phys. rev. B 70, 035319 (2004)

[13] U. Fano, Phys. Rev. 124, 1866 (1961)
[14] E. Dagotto and A. Moreo, Phys. Rev. D 31, 865 (1985); E. Gagliano, E. Dagotto, A. Moreo and F. Alcaraz, Phys. Rev. B 34, 1677 (1986).

[15] V. Ferrari, G. Chiappe, E. V.Anda, and M. A. Davidovich, Phys. Rev. Lett. 82, 5088 (1999).

[16] C. Caroli et al., J. Phys. C 4, 916 (1971); W. Metzner, Phys. Rev. B 43, 8549 (1991).

[17] T. Jamneala, V. Madhavan, and M. F. Crommie, Phys. Rev. Lett. 87, 256804 (2001).

[18] V. Savkin et al., Phys. Rev. Lett. 94, 026402 (2005)

[19] A. Aligia, preprint.

[20] Yu. B. Kudasov and V. M. Uzdin, Phys. Rev. Lett. 89, 276802 (2002).

[21] B. Lazarovits et al., Phys. rev. Lett. 95, 077202 (2005)

[22] M. Vojta, R. Bulla and W. Hofstetter, Phys. Rev. B, 65, 140405 (2002)

[23] D. Saraga and D. Loss, Phys. Rev. Lett. 90, 166803 (2003); T. Kuzmenko, K. Kikoin and Y. Avishai, cond-mat/0507488 A. Vidan et al., App. Phys. Lett. 85, 3602 (2004) 